УДК 65.018.2

\title{
СОВЕРШЕНСТВОВАНИЕ МЕТОДА АНАЛИЗА ВИДОВ И ПОСЛЕДСТВИЙ РИСКА НЕСАНКЦИОНИРОВАННОГО ОТБОРА ГАЗА
}

\author{
(c) 2021 Ю.С. Клочков ${ }^{1}$, Г.А. Фокин ${ }^{1}$, О.В. Сыровацский ${ }^{2}$ \\ ${ }^{1}$ Санкт-Петербургский политехнический университет Петра Великого, Санкт-Петербург, Россия \\ ${ }^{2} \mathrm{OOO}$ «Газпром межрегионгаз инжиниринг», Санкт-Петербург, Россия
}

Статья поступила в редакцию 16.11.2021

\begin{abstract}
B статье приводятся результаты развития метода FMEA-анализа на примере несанкционированного отбора газа. Улучшения метода основаны на классификации субъективных факторов и объективных предпосылок, которые влияют на оценку частоты возникновения, оценку вероятности обнаружения и на оценку значимости последствий при проведении анализа рисков. Кроме того, в качестве оценки влияния факторов и предпосылок использован Дом качества. Результаты, приведенные в статье, могут быть рассмотрены для других областей с соответствующим изменением классификатора, зависимостей и результатов экспертной оценки. Статья может быть интересна специалистам, применяющим FMEA в своей практической деятельности, а также аспирантам и ученым в области менеджмента качества продукции.
\end{abstract}

Ключевые слова: несанкционированный отбор газа, FMEA, приоритетное число риска.

DOI: $10.37313 / 1990-5378-2021-23-6-18-25$

\section{ВВЕДЕНИЕ}

Проблема несанкционированного отбора газа имеет государственное значение, поэтому применение передовых методов менеджмента качества для решения данной научно-практической проблемы является актуальной задачей. Данная проблема имеет системный характер и обусловлена большим количеством причин. Но, кроме причин (низкая достоверность учета расхода газа, слабый уровень защищенность газораспределительной сети и других), действуют предпосылки и факторы (например, распространенность в сети Интернет способов несанкционированного отбора газа), которые в значительной степени увеличивают рост объемов несанкционированного отбора газа.

Несанкционированный отбор газа приводит не только к потере ожидаемых денежных средств, но и является опасным нарушением, которое может повлечь за собой серьезные аварии, в том числе и с летальным исходом. Фактически для предотвращения угрозы несанкционированного отбора газа необходимо устранить уязвимости сети газораспределения.

Клочков Юрий Сергеевич, доктор технических наук, доцент, и.о. проректора по научно-организационной деятельности СПбПУ.E-mail: y.kloch@gmail.com Фокин Георгий Анатольевич, доктор технических наук, доцент, заведующий кафедрой «Газотурбинные агрегаты для газоперекачивающих станций».

Сыровацский Олег Валерьевич, начальник научнообразовательного центра ООО «Газпром межрегионгаз инжиниринг».E-mail:sov708@yandex.ru

\section{КЛАССИФИКАЦИЯ ОБЪЕКТИВНЫХ ПРЕДПОСЫЛОК И СУБЪЕКТИВНЫХ ФАКТОРОВ, ВЛИЯЮЩИХ НА ПРИОРИТЕТНОЕ ЧИСЛО РИСКА}

Как известно расчет приоритетного числа риска основан на определении оценок значимости последствий, оценок частоты возникновения и оценок сложности обнаружения [1-5]. Разработаем методику определения данных оценок, отличающуюся тем, что учитываются объективные предпосылки и субъективные факторы влияющие на риск совершения несанкционированного отбора газа.

В результате разработанной методики становится очевидным, что оценки значимости последствий, оценки частоты возникновения и оценки сложности обнаружения являются комплексными оценками и должны быть представлены научно-обоснованной системой сбора и анализа соответствующей информации.

Кроме того, при построении системы сбора информации установлено, что одни и те же предпосылки и/или факторы могут влиять не только на одну оценку, входящую в расчет приоритетного числа риска, но и на несколько сразу. То есть возникают ситуации, когда оценки значимости, частоты и обнаружения зависимы между собой в конкретной рассматриваемой системе [6-10].

В этом случае можно либо порекомендовать исключение учета единичных показателей из расчета комплексных оценок, либо приять факт зависимости оценок между собой. В любом случае процедура расчета приоритетного числа ри- 
ска должна обладать прозрачностью, достоверностью и быть эффективной [1-10].

Классификация субъективных факторов. К субъективным факторам, влияющим на риск несанкционированного отбора газа, следует относить факторы, формируемые обществом и человеком. К таким факторам относятся:

- Среднестатистическая оценка числа несанкционированного отбора газа в регионе;

Следует отметить, что если уровень несанкционированного отбора высокий, то значит, что общество настроено на поиск методов несанкционированного отбора. Фактически можно сказать, что несанкционированный отбор становится традиционным способом ведения хозяйства.

- Информационная доступность материалов, касательно методик несанкционированного отбора газа;

Для комплексной оценки риска несанкционированного отбора газа необходим анализ внешней среды с точки зрения доступности материалов, в которых раскрываются методики несанкционированного отбора газа. Наличие видео материалов, схем и подходов по вмешательству в приборы учета повышают риск несанкционированного отбора газа. Поиск такой информации, ее изъятие, установления авторов позволит сократить число нарушений. К информации, доступность к которой может повлиять на риск несанкционированного обора газа можно отнести:

- Эксплуатационная информация;

- Информация, касающаяся сроках и интервалах контроля;

- Информация о методах контроля;

- Данные об уязвимостях;

- Информация о программном обеспечении.

• Уровень компетенций нарушителей;

Данный фактор влияет на выбор средств учета расхода газа. Чем выше будет требоваться компетенция нарушителя, тем меньше экономическая целесообразность нарушения и, тем меньше число потенциальных нарушителей.

Следует отметить, что субъективные факторы должны постоянно пересматриваться и дополняться.

Объективные предпосылки. К объективным предпосылкам следует отнести технические и социально-экономические условия.

-Уровень доходов в рассматриваемом регионе;

Если стоимость газа по сравнению с уровнем дохода является слишком высокой, то риск несанкционированного отбора будет возрастать.

- Размер штрафа;

Если уровень штрафа ниже, чем получаемая прибыль от несанкционированного расхода газа, то риск нарушения очень высок.

- Средства реализации несанкционированного отбора газа;
Нарушитель либо использует доступные в свободной продаже технические средства и программное обеспечение, либо специально разработанные технические средства и программное обеспечение. Необходима оценка стоимости данных средств и возможности их применения.

- Достоверность и независимость процедуры контроля.

Чем выше достоверность и независимость, тем ниже риск несанкционированного отбора газа.

Следует отметить, что объективные предпосылки должны постоянно пересматриваться и дополняться.

Оценка возможности обнаружения. На данную оценку непосредственно оказывают влияние:

- Достоверность и независимость процедуры контроля;

В случае если уровень достоверности контроля недостаточный, то обнаружить несанкционированный отбор газа становится невозможным. Так же в случае, если не обеспечена независимость процедуры контроля, то несанкционированный отбор газа обнаружен не будет.

Чем ниже достоверность и независимость контроля, тем выше риск того, что несанкционированный отбор газа будет не обнаружен, поэтому чем ниже показатели достоверности и независимости, тем выше баллы оценки возможности обнаружения.

- Уровень компетенций нарушителей.

Чем выше уровень компетенции нарушителя, тем выше должная быть баллы оценки возможности обнаружения.

Косвенное влияние:

- Информационная доступность материалов, касательно методик несанкционированного отбора газа;

Чем выше информационная доступность материалов, касающихся методик несанкционированного отбора газа тем выше балл оценки возможности обнаружения.

Чем выше доступность методик несанкционированного отбора, тем выше риск того, что обнаружить нарушение в счетчиках газа или в сети будет сложнее. Таким образом методики несанкционированного отбора, которые, например, представлены в сети Интернет увеличивают риск того, что несанкционированный отбор газа не будет обнаружен. Остальные оценки строятся аналогично.

\section{МЕТОДИКА РАСЧЕТА ПРИОРИТЕТНОГО ЧИСЛА РИСКА, УЧИТЫВАЮЩАЯ РЕЗУЛЬТАТЫ КЛАССИФИКАЦИИ ОБЪЕКТИВНЫХ ПРЕДПОСЫЛОК И СУБЪЕКТИВНЫХ ФАКТОРОВ}

Расчет приоритетного числа риска основан на анализе значимости последствий потенциальных несоответствий, оценки вероятности возникнове- 


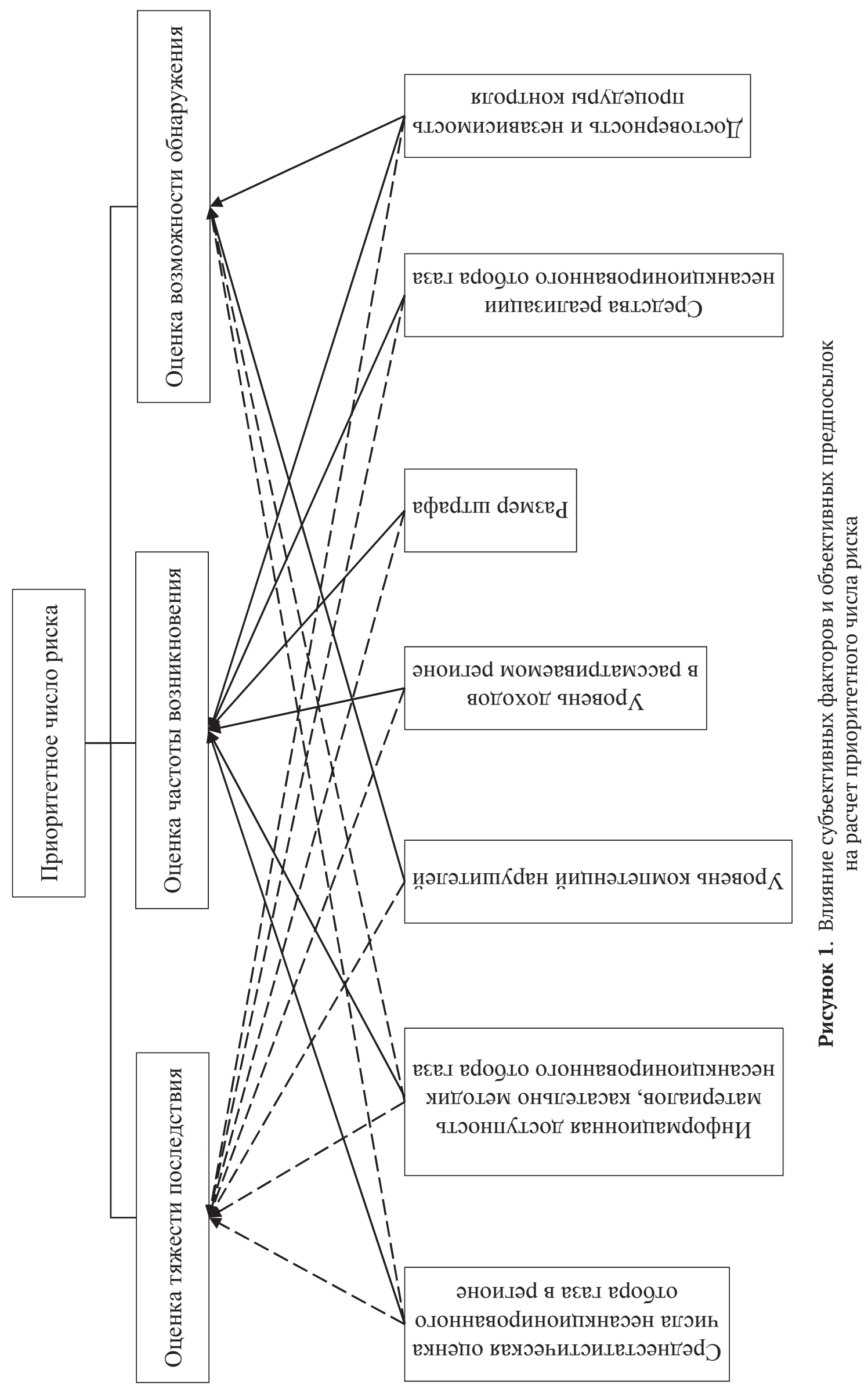




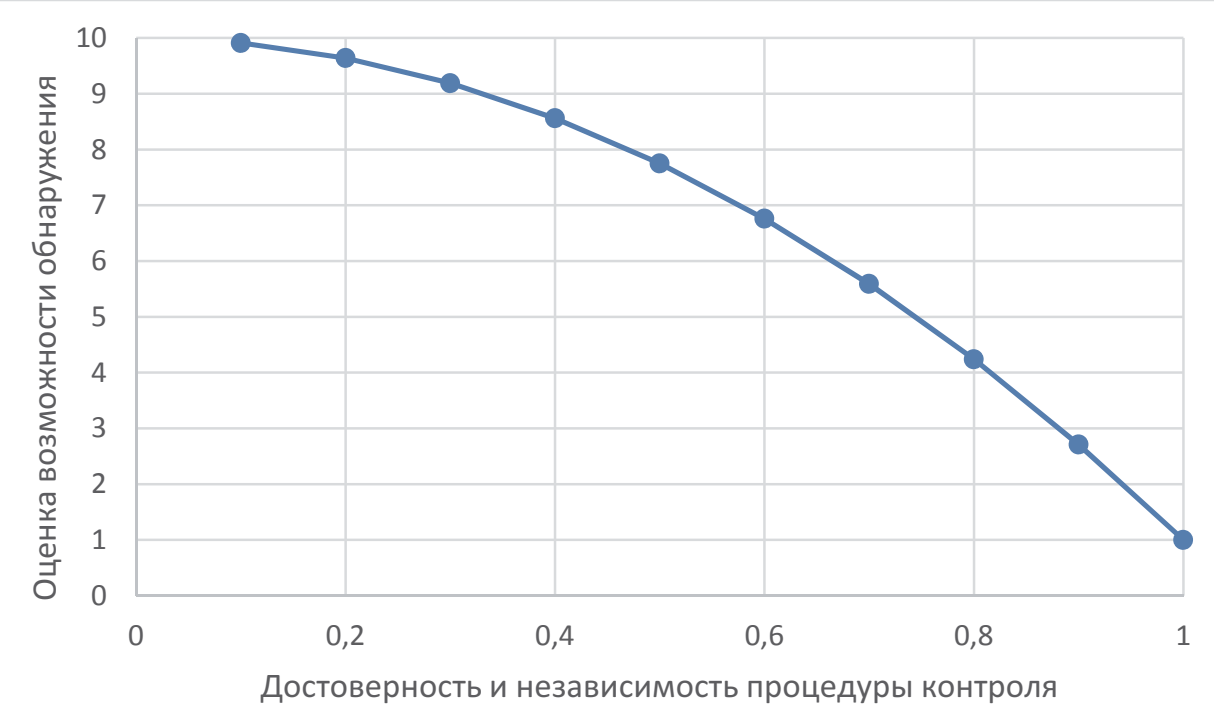

Рисунок 2. Предлагаемая модель оценки возможности обнаружения относительно достоверности и независимости процедуры контроля

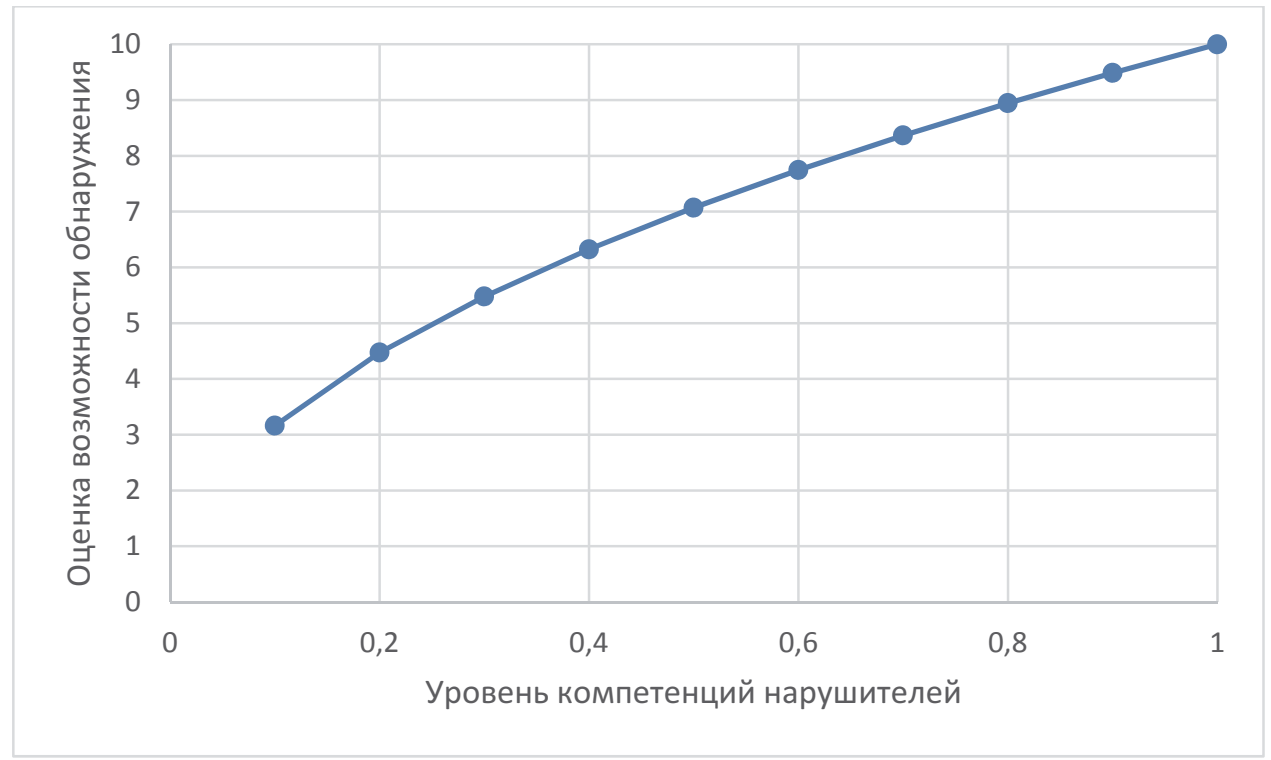

Рисунок 3. Предлагаемая модель оценки возможности обнаружения относительно уровня компетенции нарушителя

ния несоответствия, причины или последствия, а также анализа вероятности обнаружения несоответствия, причины или последствия.

В нашем примере рассмотрены такие факторы и предпосылки:

- достоверность и независимость процедуры контроля;

- средства реализации несанкционированного отбора газа;

- размер штрафа;

-уровень доходов в рассматриваемом регионе;

- уровень компетенций нарушителей;

- информационная доступность материалов, касательно методик несанкционированного отбора газа;
- среднестатистическая оценка числа несанкционированного отбора газа в регионе.

Выше определены модели влияния данных факторов на значения частоты возникновения и возможности обнаружения. Кроме того, как показано на рисунке 2.1 известно, что на оценки частоты возникновения и возможности обнаружения влияют несколько факторов и предпосылок, имеется прямое и косвенное влияние. Для того чтобы решить проблему итогового определения значений частоты возникновения и возможности обнаружения предложим воспользоваться известной методологией QFD-анализа. Для этого построим Дом качества, в котором требования будут представлены входящими в расчет при- 


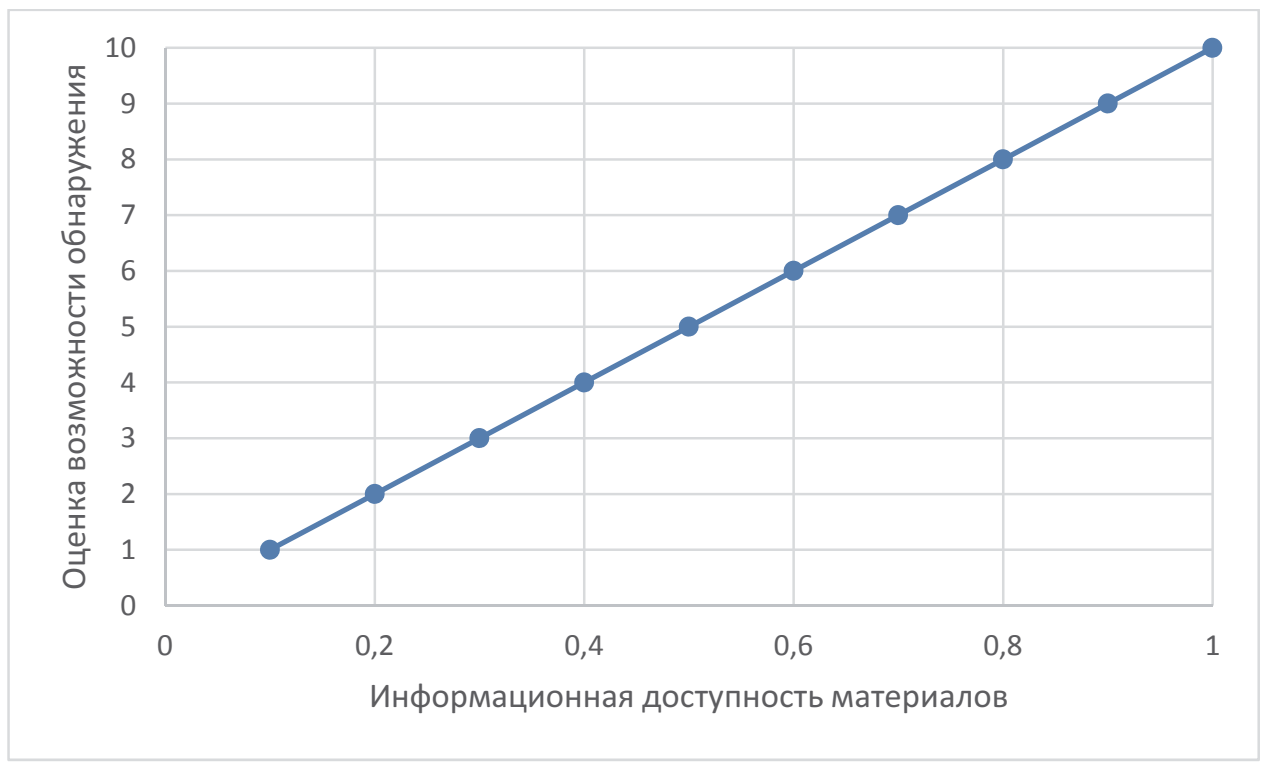

Рисунок 4. Предлагаемая модель оценки возможности обнаружения относительно информационной доступности материалов

оритетного числа риска показатели (значимость последствий, оценка частоты возникновения и оценка возможности обнаружения).

Допустим, что для определения величины оценки возможности обнаружения было установлено что:

1. Конкретная ситуация с «Достоверностью и независимостью процедуры контроля» такова, что по рисунку 2 экспертная группа предложила установить значение Оценки возможности обнаружения равным 5;

2. Конкретная ситуация с «Уровнем компетенций нарушителей» такова, что по рисунку 3 экспертная группа предложила установить значение Оценки возможности обнаружения равным 7;

3. Конкретная ситуация с «Информационной доступностью материалов, касательно методик несанкционированного отбора газа» такова, что по рисунку 4 экспертная группа предложила установить значение Оценки возможности обнаружения равным 6;

4. Конкретная ситуация со «Среднестатистической оценкой числа несанкционированного отбора газа в регионе» такова, что экспертная группа предложила установить значение Оценки возможности обнаружения равным 4.

Тогда для определения итогового значения величины оценки возможности обнаружения воспользуемся Домом качества, в котором установлены проценты влияния, указанных факторов и предпосылок

Следует отметить, что данный пример показывает возникновение конфликта экспертных оценок, который заключается в следующем: на рисунке 1 установлено, что только четыре фактора и предпосылки влияют на возможность обнаружения, но из Дома качества видно, что таких факторов и предпосылок шесть. При воз- никновении подобных конфликтов экспертных оценок необходимо установить модели влияния дополнительных факторов и предпосылок на расчет показателя FMEA.

После выполнения данной процедуры получаем оценки двух других факторов и предпосылок. Например, конкретная ситуация со «Средствами реализации несанкционированного отбора газа» говорит, что оценки возможности обнаружения должна быть равной 4, а конкретная ситуация с «Уровнем доходов в рассматриваемом регионе» говорит, что оценки возможности обнаружения должна быть равной 3. Расчет оценки возможности обнаружения сведем в таблицу 2.

Итоговый расчет значения оценки возможности обнаружения сводится к сумме произведений соответствующих столбцов таблицы.

Кроме подобной модели расчета можно предложить выбирать максимум из предложенных экспертами оценок, тогда итоговое значение оценки возможности обнаружения будет равно 7.

Дальнейший расчет оценки вероятности возникновения ведется аналогичным образом, определения приоритетного числа риска в данном случае не отличается от принятых моделей и осуществляется за счет перемножения оценок значимости последствий, частоты возникновения и возможности обнаружения.

\section{МЕТОДИКА УЧЕТА ВЗАИМОСВЯЗИ ФАКТОРОВ И ПРЕДПОСЫЛОК}

При работе с экспертными группами часто обнаруживается взаимосвязь между факторами и предпосылками. В случаях, когда необходимо учесть такую взаимосвязь разработана дан- 


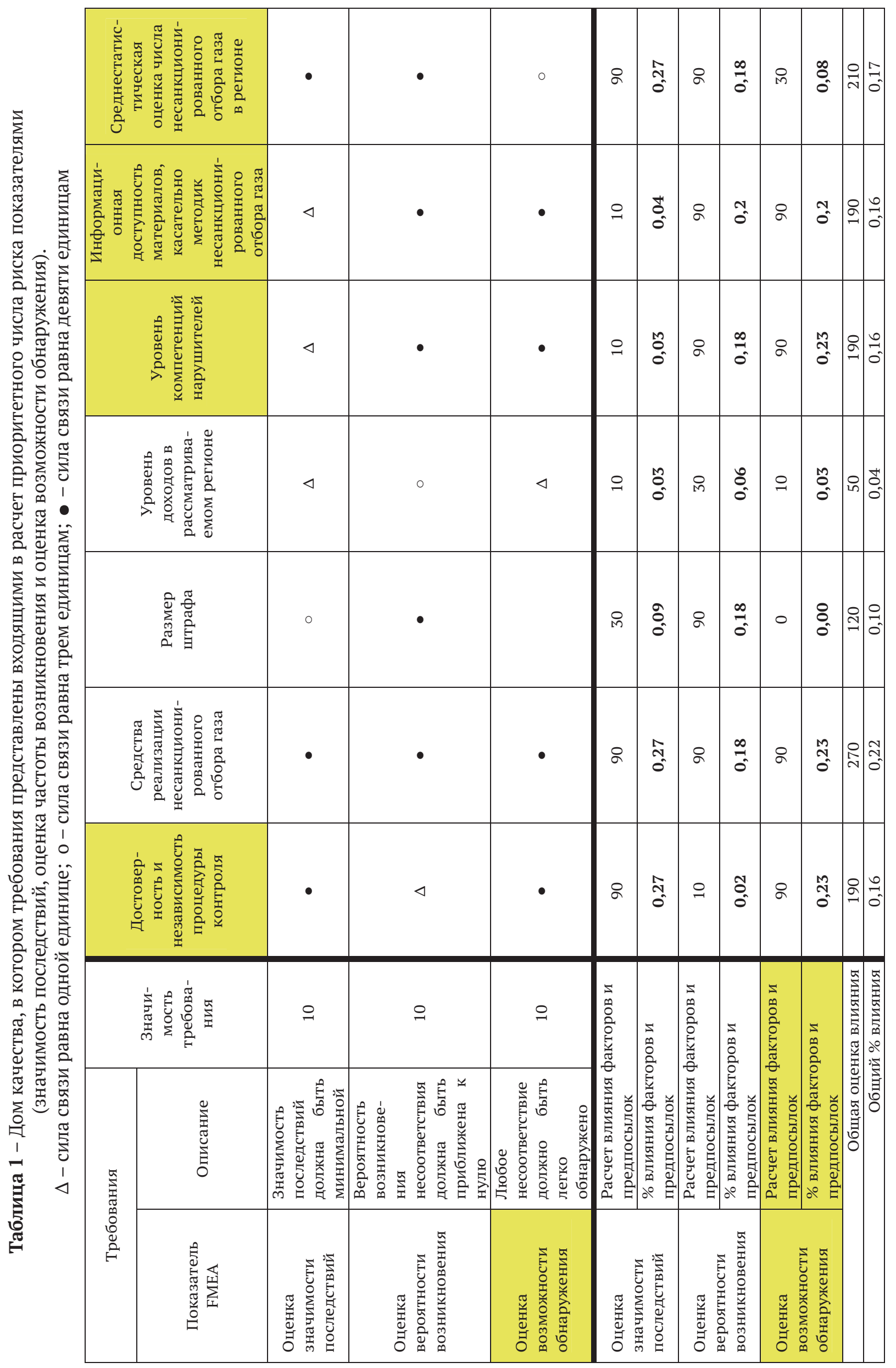


Таблица 2 - Расчет оценки возможности обнаружения несанкционированного отбора газа

\begin{tabular}{|c|c|c|c|c|c|c|}
\hline $\begin{array}{c}\text { Факто- } \\
\text { ры и } \\
\text { предпо- } \\
\text { сылки }\end{array}$ & $\begin{array}{c}\text { Досто- } \\
\text { верность и } \\
\text { независи- } \\
\text { мость } \\
\text { проце- } \\
\text { дуры } \\
\text { контроля }\end{array}$ & $\begin{array}{c}\text { Средства } \\
\text { реализации } \\
\text { несанкциони- } \\
\text { рованного } \\
\text { отбора газа }\end{array}$ & $\begin{array}{c}\text { Уровень } \\
\text { доходов } \\
\text { в рассмат- } \\
\text { риваемом } \\
\text { регионе }\end{array}$ & $\begin{array}{c}\text { Уровень } \\
\text { компе- } \\
\text { тенций } \\
\text { наруши- } \\
\text { телей }\end{array}$ & $\begin{array}{c}\text { Информацион- } \\
\text { ная } \\
\text { доступность } \\
\text { материалов, } \\
\text { касательно } \\
\text { методик } \\
\text { несанкциони- } \\
\text { рованного } \\
\text { отбора газа } \\
\end{array}$ & $\begin{array}{c}\text { Среднестатисти- } \\
\text { ческая оценка } \\
\text { числа } \\
\text { несанкциониро- } \\
\text { ванного отбора } \\
\text { газа в регионе }\end{array}$ \\
\hline $\begin{array}{c}\text { Эксперт } \\
\text { ные } \\
\text { оценки }\end{array}$ & 5 & 4 & 3 & 7 & 6 & 4 \\
\hline $\begin{array}{c}\% \\
\text { влияни } \\
\text { я } \\
\text { фактор } \\
\text { ов и } \\
\text { предпос } \\
\text { ылок }\end{array}$ & 0,23 & 0,23 & 0,03 & 0,23 & 0,2 & 0,08 \\
\hline \multicolumn{6}{|c|}{ Итоговое значение оценки возможности обнаружения } & 5,29 \\
\hline
\end{tabular}

ная методика перерасчета влияния факторов и предпосылок на оценки тяжести последствий, возможности обнаружения и частоты возникновения. Допустим экспертами обнаружена следующая взаимосвязь (табл. 3).

Тогда для расчета влияния факторов и предпосылок предложим следующую формулу:

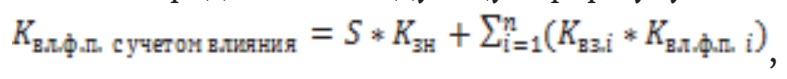

где $S$ - сила связи;

$K_{\text {зн }}-$ значимость требований;

$K_{\text {вз.і }}$ - степень взаимосвязи между факторами и предпосылками;

$K_{\text {в.,ф... i }}-$ расчет влияния факторов и предпосылок.

В результате появляется возможным учитывать взаимосвязь факторов и предпосылок при расчете приоритетного числа риска, что позволяет проводить комплексный учет экспертных мнений и оценок и делает результаты исследования максимально точными и достоверными.

\section{ОБЩИЕ ВЫВОДЫ ПО СТАТЬЕ}

Установлено, что для повышения эффективности процедуры анализа видов и последствий несанкционированного отбора газа необходимо провести классификацию объективных предпосылок и субъективных факторов, которые влияют на результаты расчета приоритетного числа риска;

Предложен метод расчета приоритетного числа риска, учитывающий внешние по отношению к рассматриваемому объекту (например, газораспределительной сети) FMEA-анализа со-

Таблица 3 - Пример взаимосвязи факторов и предпосылок

\begin{tabular}{|c|c|c|c|c|c|c|c|c|}
\hline & 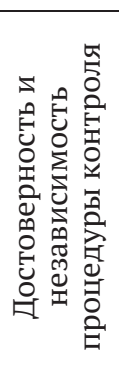 & 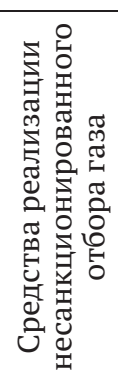 & 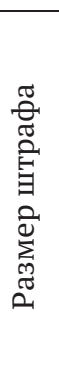 & 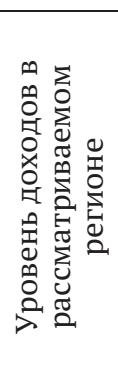 & 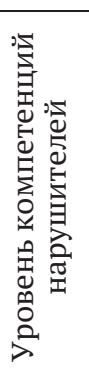 & 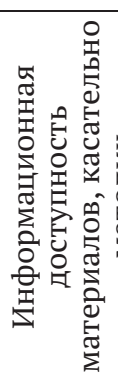 & 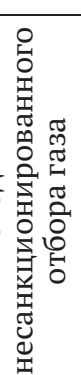 & 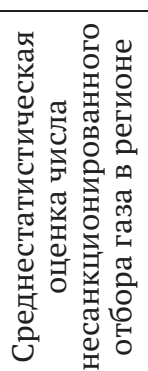 \\
\hline $\begin{array}{c}\text { Информационная } \\
\text { доступность } \\
\text { материалов, } \\
\text { касательно методик } \\
\text { несанкционированного } \\
\text { отбора газа } \\
\end{array}$ & - & 0,28 & - & - & 0,38 & - & & 0,34 \\
\hline
\end{tabular}


бытия (объективные предпосылки и субъективные факторы), которые влияют на последствия, причины и несоответствия (отказы);

Предложен механизм учета взаимосвязи объективных предпосылок и субъективных факторов, что позволило повысить объективность результатов анализа риска.

\section{СПИСОК ЛИТЕРАТУРЫ}

1. Tsai, S., Zhou, J., Gao, Y., Wang, J., Li, G., Zheng, Y., Xu, $W$. (2017). Combining FMEA with DEMATEL models to solve production process problems // PLOS ONE, 12(8) doi:10.1371/journal.pone.0183634

2. Wan, C., Yan, X., Zhang, D., Qu, Z., \& Yang, Z. (2019). An advanced fuzzy bayesian-based FMEA approach for assessing maritime supply chain risks // Transportation Research Part E: Logistics and Transportation Review, 125, 222-240. doi:10.1016/j. tre.2019.03.011

3. Zarei, E., Azadeh, A., Khakzad, N., Aliabadi, M. M., \& Mohammadfam, I. (2017). Dynamic safety assessment of natural gas stations using bayesian network // Journal of Hazardous Materials, 321, 830-840. doi:10.1016/j.jhazmat.2016.09.074

4. Zhang, H., Dong, Y., Palomares-Carrascosa, I., \& Zhou, H. (2019). Failure mode and effect analysis in a linguistic context: A consensus-based multiattribute group decision-making approach // IEEE Transactions on Reliability, 68(2), 566-582. doi:10.1109/TR.2018.2869787

5. Антипов, Д. В. Совершенствование метода FMEA -анализа для оценки рисков / Д. В. Антипов, В. А. Буркова // Дневник науки. - 2019. - № 5(29). - С. 79.

6. Панюков, В. Н. Моделирование процедуры FMEA с ИТ-поддержкой в нотации BPMN / В. Н. Панюков, В. Н. Козловский, Д. В. Айдаров // Методы менеджмента качества. - 2019. - № 12. - С. 28-35.

7. Панюков, Д. И. Моделирование процедуры FMEA: анализ рисков / Д. И. Панюков, В. Н. Козловский, Д. В. Айдаров // Методы менеджмента качества. 2019. - № 9. - С. 34-43.

8. Панюков, Д. И. Моделирование процедуры FMEA: методология и стратегия / Д. И. Панюков, В. Н. Козловский, Д. В. Айдаров // Методы менеджмента качества. - 2019. - № 7. - С. 30-38.

9. Панюков, Д. И. Модель системы интеллектуальной помощи при использовании поддержки FMEA. Часть 2 на потребительском рынке / Д. И. Панюков, В. Н. Козловский // Качество и жизнь. - 2021. - № 3(31). C. 8-18. - DOI 10.34214/2312-5209-2021-31-3-8-18.

10. Панюков, Д. И. Новое руководство по FMEA: анализ отказов процессов / Д. И. Панюков, В. Н. Козловский, Д. И. Благовещенский // Методы менеджмента качества. - 2020. - № 12. - С. 30-36.

\title{
IMPROVEMENT OF FAILURE MODE AND EFFECTS ANALYSIS METHOD FOR UNAUTHORIZED GAS TAPPING
}

\author{
(c) 2021 Yu.S. Klochkov' ${ }^{1}$, G.A. Fokin ${ }^{1}$, O.V. Syrovatskiy² \\ ${ }^{1}$ Peter the Great St. Petersburg Polytechnic University (SPbPU), St. Petersburg, Russia \\ ${ }^{2}$ Gazprom Mezhregiongaz Engineering, St. Petersburg, Russia
}

\begin{abstract}
The article presents the results of developing FMEA method on an example of illegal gas-tapping. The method was improved by means of classifying subjective factors and objective preconditions, which influence the estimates of the rate of occurrence, detection probability and significance of consequences in risk analysis. In addition, we used Quality Function Deployment to assess the influence of the factors and preconditions. The results presented in the article can be applicable in other areas with a corresponding change in the classifier, dependences and expert evaluation results. The paper is of interest for specialists applying FMEA in practice, as well as for postgraduate students and product quality scientists.
\end{abstract}

Keywords: illegal gas-tapping, FMEA, Risk Priority Number

DOI: $10.37313 / 1990-5378-2021-23-6-18-25$

\footnotetext{
$\overline{\text { Yury Klochkov, Doctor of Technical Sciences, Associate }}$ Professor, Acting Vice-Rector for Scientific-Organizational Activities of SPbPU.E-mail: y.kloch@gmail.com

Georgy Fokin, Doctor of Technical Sciences, Associate Professor. Head of the Department «Gas Turbine Units for Gas Pumping Stations».

Oleg Syrovatskiy, Head of Academic Center of LLC Gazprom MezhregiongazEngineering.E-mail:sov708@yandex.ru
} 